\title{
Unveiling the Structure: Effects of Social Feedback on Communication Activity in Online Multiplayer Videogames
}

\author{
Luciano Gamberini, Francesco Martino, Fabiola Scarpetta, Andrea Spoto, \\ and Anna Spagnolli \\ Human Technology Lab \\ Department of General Psychology \\ University of Padova - Italy \\ \{luciano.gamberini, andreaspoto, fabiola.scarpetta\} @gmail.com \\ \{francesco.martino, anna.spagnolli\}@unipd.it
}

\begin{abstract}
Feedback intervention in computer-mediated situations can be interpreted as a way to augment communication. According to this idea, this study investigates the effect of providing a group with a Social Network Analysis-based feedback on communication in an on-line game where players talk to each other via textual chat. Three different situations across two different sessions were compared: an Informed Group with a correct feedback, a notInformed Group with no feedback and a mis-Informed group with an incorrect feedback. Results show that giving correct information increases the related dimensions of communication, while the absence of feedback and the incorrect feedback were not accompanied by any significant modification.
\end{abstract}

Keywords: social network analysis, feedback, augmented communication, cooperative online game.

\section{Introduction}

Feedback is a well known resource for increasing people's motivation and performance, as highlighted by several models such as Cybernetic or Control Theory [1], Goal-Setting Theory [2] and Social Learning Theory [3]. When feedback is provided intentionally by an agent different from the one performing the activity, and consists of information that is not the spontaneous byproduct of the ongoing task, then we have a special kind of procedure called Feedback Intervention [4]. It is defined as a series of "actions, taken by (an) external agent(s) to provide information regarding some aspect(s) of one's task performance (p. 255)". By extending this definition to other processes than just 'performance' and including technology as one possible 'external agent' providing the feedback, feedback intervention emerges as a strategy of augmented interaction. Information on the quality or quantity of interaction is provided to the users by way of technical devices incorporated in the mediated environment where the activity takes place. In this case, technology is employed to enrich the communicative process with cues that would not be offered by the original 
setting [as in 5, 6], instead of just trying to imitate the characteristic of face-to-face communication.

This study presented here is part of an EU-funded Integrated Project called 'PASION' (Psychologically Augmented Social Interaction Over Networks) [7], aiming at augmenting mediated interaction by making more evident the status of the larger social network to which the individuals contribute with their actions. This first experiment investigates the usage of feedback based on Social Network Analysis (SNA) [8] and its effects on the communicational structure of the users.

\subsection{Feedback Effectiveness}

Different studies have observed the way in which feedback on a certain dimension of group-mediated activity is able to affect users' behavior. Losada, Sanchez and Noble gave their participant a complex feedback about collaborative exchanges in two different tasks (a list ranking task and an in-basket simulation) with or without a computer-supported collaborative environment. They found that feedback was able to reverse the difference in the socio-emotional behavior produced in mediated and nonmediated environments: this behavior tended to decrease in mediated environments, except when a feedback was provided [9]. Di Micco, Pandolfo and Bender [10] used a display during an information-sharing task to illustrate the users' participation rates. They found that in some groups the participation rate changed in a direction connected with the evaluation implicit in the feedback. Zumbach, Schönemann and Reimann (2005) studied a problem-solving task executed by dyads through an HTML-based collaborative system. Each episode of collaborative behaviors was detected by a human observer, and immediately displayed on the participants' monitors, along with an appraisal. The task type (homogeneous versus distributed resources) and the provision of feedback (with/without) were manipulated. The highest amount of collaborative events was detected in the condition with distributed resources and with feedback [11].

These three studies converge on the result that feedback presentation is able to produce a change in those aspects of the performance that are covered by the feedback. However, they also suggest the need to pay attention to the nature of the feedback provided, for instance its accuracy [10] and complexity [9]. Classic psychological studies on feedback offer some specific recommendations in this respect. Ilgen, Fisher and Taylor in their review underline the role of credibility [12]. Kluger and DeNisi, on the basis of an extensive meta-analysis of 131 papers, suggested that feedback works by drawing user's attention to one of three levels: taskmotivation, task-learning and meta-task (self). When both goal and feedback are clear, optimal usage of a feedback occurs when the processing remains on the taskmotivation level, with the user trying to find a way to fill the standard-feedback gap. Finally, they suggested that feedback providing specific and detailed information on the way in which the task must be executed facilitates learning insofar as it is congruent with the task and helps reject erroneous hypotheses. In the case of a collaborative tasks, feedback at a group level may provide that kind of congruent information that increases the overall performance, since the individual contributions would become more precise, and appropriate to the actual status of the activity. To this respect, there is some empirical evidence that the best feedback is able to link the 
individual actions to the group status without separating the two kinds of information. Zander and Wolfe [13] found that individual and group feedback together were more effective than either feedback alone. DeShon, Kozlowsi, Shmidt, Milner and Wiechman [14] were interested in tasks requiring both individual and team effort allocation ('discretionary tasks'), in the assumption that they represent the most recurrent form of tasks in real-life groups. They found that the highest level of individual and team-oriented performance occurred when team members received a single, focused source of feedback, but stressed that the feedback they provided did not allow to relate the individual performance to the team one, and this prevented participants from knowing how to use the two kinds of information.

In the present study, we resorted to an SNA-based feedback, which is able to position the individual user within the group structure and dynamics. SNA is a formal theory based on networks of relations among social actors [8]: social actors are treated as nodes in the network, and relations among them are represented as ties or links. SNA can provide valuable tools to describe, evaluate, and visualize the quantity and quality of social relations, and for this reason it has been applied to the analysis of work organizations [15], and, recently, to on-line communication $[16,17,18,19]$. Some research in particular focuses on the use of SNA feedback in mediated environments. Isbell, Kearns, Singh, Shelton \& Stone (2006) showed that a 'bot' with the ability to give social dyadic relation statistics (e.g. "who loves me", and so on) and provide social ranking increased social participation in the LambdaMoo in which it was introduced [20]. Morris [21] found a way to increase elderly people's self-awareness and confidence in the possibility of improving their social life by way of a socialnetwork display to control their own social activity. Authors found that participants were able to draw attention to the part of the network with fewer contacts, that they were able to cope with this problem and that they were more socially active.

Our goal with the study presented here is to devise an experiment in order to test specifically whether providing SNA indexes to people involved in a group task is able to change their behavior and to do it in a way related to the content of the feedback.

\section{Method}

To investigate a common form of a mediated collaborative task, we used an on-line treasure-hunt game, built with the open-source cooperative multiplayer graphical game called 'Crossfire' [22]. Eight participants were assigned a PC station and an avatar each, with which they could operate in the same digital environment. They could talk to each other exclusively via Skype ${ }^{\circledR}$ textual chat (figure 1). The aim of the game was for the group to find and pick up seven special objects hidden in the environment during a forty-minute game session. In addition to the hunt, participants had to keep their avatars alive by picking up some food placed in the game environment.

Simple commands were implemented: the four directional arrows moved the avatars, the "a" keystroke picked up objects or allowed one to enter/exit buildings, the left button of the mouse highlighted the name of other avatars actually present in the screen, and the right button of the mouse picked up the special objects. Players were 


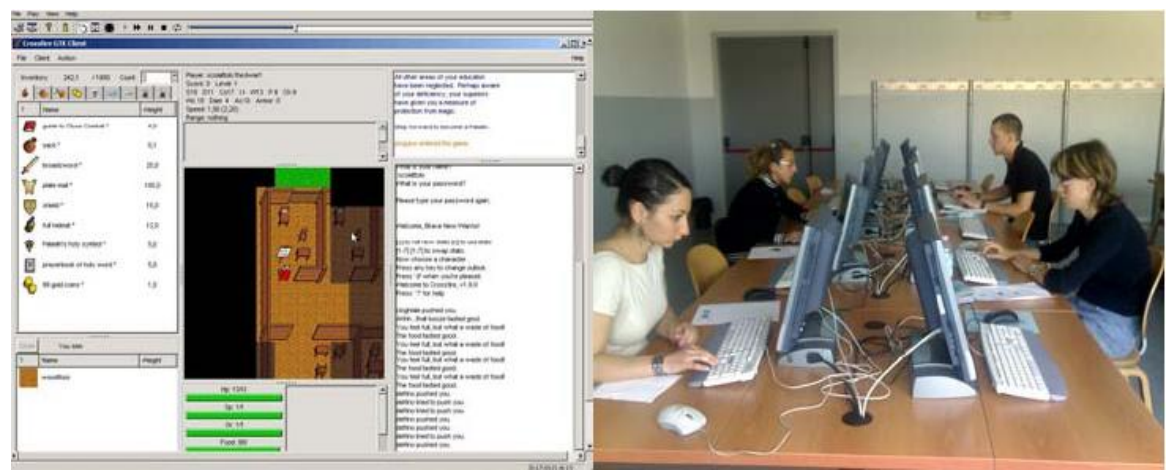

Fig. 1. A screenshot from the game interface (left) and a picture of the game session (right)

instructed to make use of the cues spread across the environment containing some directions to the 'treasures', and to communicate; they were made aware of the large extension of the environment (too large to be explored alone in 40 minutes) and that the score was calculated on the group as a whole.

\subsection{Participants}

Participants were twenty-four Psychology students at the University of Padova aged 24,88 on average (standard deviation 2.96). The proportion of males and females in all groups was the same (one male every three females). All participants were accustomed to using computers and instant messaging software but none of them was an expert videogame user.

\subsection{Procedure}

Participants played during two game sessions a week apart. The first session was preceded by a five minutes practice to get familiar with the commands, the game rules and the digital environment. In the two sessions, the game scenarios were different, but had the same extension. Also, the special objects were distributed in different places, but with the same ratio. In order to avoid biases due to participants' previous relationships, each participant was assigned a nick name by the experimenters. Players were requested not to reveal their avatars' identity until the end of the whole study. A member of the research team supervised each session to warrant its regularity.

\subsection{Experimental Design}

To test the effect of SNA feedback on participants' activity, a two-factor experimental design was applied, with a two-level between-subjects factor called "feedback" ('Informed' versus 'not-Informed' groups) and a two-level within-subjects factor called "session". The feedback consisted of histograms representing the results of the SNA conducted on the first session data, and was provided to participants in the "with feedback' conditions a few minutes before the beginning of the second session. 
In addition, to make sure that it was not just the provision of a feedback to cause an effect regardless of the feedback content, we introduced a third condition in which participants were provided with a false feedback ('Misinformed' group). The data of this group were analyzed separately because of its late introduction in the design.

\subsection{Data Collection and Feedback Construction}

The data collected were the log files of each dyadic conversation occurring within the game session in the instant messaging software. From the chat logs we identified two different kinds of information used to calculate SNA indexes. The first data were the thread-starting requests, namely messages starting a thread of subsequent messages on the same topic. This measure is boolean, from $A$ to $B(A r B=1$, else $A r B=0)$, or from $\mathrm{B}$ to $\mathrm{A}(\mathrm{BrA}=1$, else $\mathrm{BrA}=0)$. The second data from the chat logs were the number of messages sent and received by each participant.

On the basis of these data we built two different types of matrices in order to perform a SNA. With the "thread starting requests" we filled in a Boolean matrix for each session, composed of eight rows and eight columns; the number of messages was inserted in valued matrices with 8 rows and 8 columns for each session. On these two types of matrices, Ucinet software [23] calculated two kinds of indexes, 'dyadbased reciprocity' and 'degree-centrality' respectively. The former represents the mutuality of choices in a network, and ranges from 0 to 1 , the latter represents the amount of communication received by a single person in the network.

Centrality and reciprocity of each player were the kind of feedback returned to the Informed and Mis-Informed group. No feedback on performance was given.

\section{Data Analysis and Results}

\subsection{Results}

In order to test the effect of the feedback on the communicational behavior we performed an ANOVA for mixed models to verify the difference between and within the Informed and the Not-informed group in the number of messages received by each participant. The ANOVA performed on the valued matrices shows that there is a significant increase of messages received by participants in the Informed Group between the first and the second session $\left(\mathrm{F}_{2,14}=8.5 ; \mathrm{p}<.05\right)$. In figure 2 , the trends of the in-degree values in the two sessions for the informed versus not informed group are shown.

Two t-tests for paired samples have been performed on the data from the Boolean matrix in order to test the difference in reciprocity between the two sessions. We found that there is a significant difference between the two sessions in the Informed group $\left(\mathrm{t}_{7}=2.85 ; \mathrm{p}<.05\right)$ while there is no significant difference in the Not-Informed group. Figure 3 shows the trends of reciprocity in the two groups under analysis.

A particular non-parametric t-test [24] has been performed to test the significance of the difference between first and second sessions in the three groups (Informed, Not-Informed and Mis-Informed). The technique used to carry out this kind of analysis is the 'bootstrap', a very powerful kind of analysis that mixes together the SNA indexes and the more traditional statistical inferential methods. The test was 


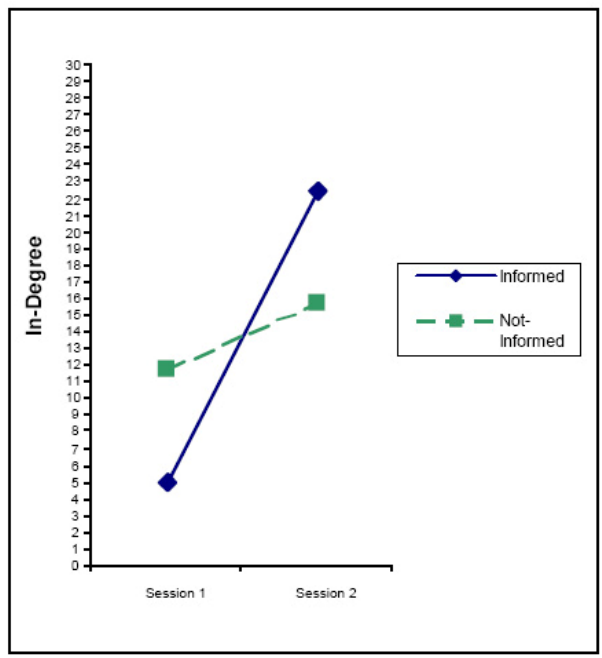

Fig. 2. Trends in in-degree centrality in the Informed and Not-Informed groups in the first and the second session

performed on density values and indicates that there was a significant difference between density of the two sessions for the Informed group $\left(t_{14}=3.46 ; p<.01\right)$ while there is no difference for the Not-Informed and the Misinformed group.

As to performance, the Informed group showed a $150 \%$ increase in the number of goblets found; the Not-Informed group showed a $20 \%$ increase; the Misinformed group presents a $20 \%$ decrease. This data suggests that providing correct feedback on the communication flow could have improved performance. However, further research is needed to confirm this interpretation.

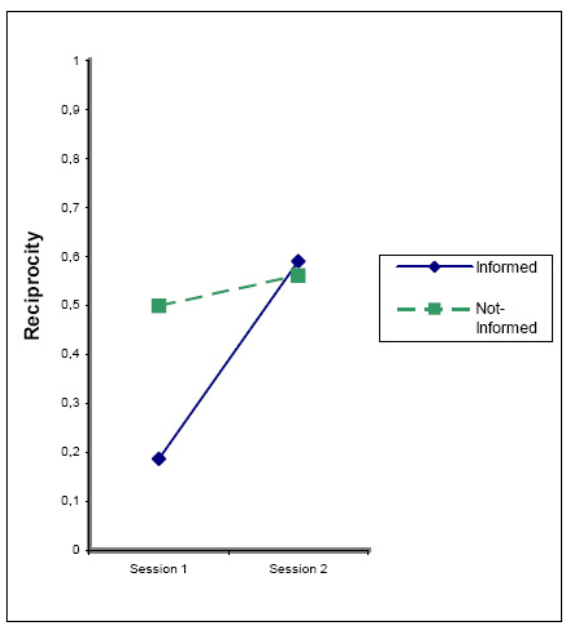

Fig. 3. Trends in dyad-based reciprocity among the Informed and Not-Informed groups in the first and the second session 


\section{Discussion and Conclusions}

The analysis showed that the social feedback on density and reciprocity provided to a network of people is accompanied by an increase in the flow of communication in the network. Not only did feedback have a decisive influence on the quantity of communication acts (density and degree-centrality), but also on the organization of the communication, with an improved symmetry in the so-called 'thread-starting requests' (reciprocity). This means that the increase was not due to practice alone since all groups underwent two game sessions, but only the Informed one showed an increased communication flow in the second one. If all members of the informed group in our study increased and improved their communication, then this may imply that the reported dimension was perceived as relevant to the task, and the presentation of the scores was perceived as an encouragement to improve on that dimensions. On the contrary, the incorrect feedback was not able to change communication activity in a significant way; this result is consistent with some previous research [10] where the feedback perceived as inaccurate was not able to trigger any change in the participants' behavior. This means that participants could make a distinction between accurate and inaccurate feedback, which has to be taken into account when planning to implement a system to augment communication.

Another interesting result was the variation of performance. The intensification of the communication flow in the Informed Group did not interfere with its performance, since the number of goblets found had a dramatic increase absent in the other conditions. The reason for this improvement, however, is not clear. It may be an effect of the strict connection between communication and performance in this specific kind of task, or a consequence of a halo effect of the feedback, which may have affected several behaviors relevant to the task.

We can conclude by saying that showing SNA indexes of communication can provide a means for community building, in the sense that increasing the awareness of the individual's impact on the group dynamics may be a tacit yet effective encouragement to intensify the connections among them.

Acknowledgments. The study was made possible by the EU funding of the PASION (Psychologically Augmented Social Interaction Over Networks) Project, FP6 - IST program - reference number 27654 - scheduled to run for four years (January 2006 December 2009).

\section{References}

1. Carver, C.S., Scheier, M.F.: Attention and self regulation: A control theory to human behavior. Springer-Verlag, New York (1981)

2. Locke, E.A., Latham, G.P.: A theory of goal setting and task performance. Prentice Hall, Englewood Cliffs, NJ (1990)

3. Bandura, A.: Social learning theory of aggression. Journal of Communication, 28(3) (1978)

4. Kluger, A.N., DeNisi, A.: The effects of feedback interventions on performance: A historical review, a meta-analysis, and a preliminary feedback intervention theory. Psychological Bulletin 119(2), 254-284 (1996) 
5. Lee, J., Jun, S., Forlizzi, J., Hudson, S.E.: New forms of interaction: Using kinetic typography to convey emotion in text-based interpersonal communication. In: Proceedings of 6th ACM conference on Designing Interactive systems (2006)

6. Danninger, M., Vertegaal, R., Siewiorek, D.P., Mamuji, A.: Using social geometry to manage interruptions and co-worker attention in office environments. In: Proceedings of, Conference on Graphics Interface, ACM International Conference Proceeding Series, vol. 112, pp. 211-218 (2005)

7. Brugnoli, M.C., Morabito, F., Walker, R., Davide, F.: The PASION Project: Psychologically Augmented Social Interaction Over Networks. PsychNology Journal 4(1), 103-116 (2006)

8. Wasserman, S., Faust, K.: Social Network Analysis. Methods and Applications. Cambridge University Press, Cambridge, MA (1994)

9. Losada, M., Sanchez, P., Noble, E.E.: Collaborative technology and group process feedback: Their impact on interactive sequences in meetings. In: Proceedings of the ACM Conference on Computer-Supported Cooperative Work, pp. 53-64 (1990)

10. DiMicco, J.M., Pandolfo, A., Bender, W.: Influencing group participation with a shared display. In: Proceedings of the, ACM Conference on Computer Supported Cooperative Work, pp. 614-623 (2004)

11. Zumbach, J., Schonemann, J., Reimann, P.: Analyzing and supporting collaboration in cooperative computer-mediated communication. In: Proceedings of the 2005 Conference on Computer Support for Collaborative Learning. International Society of the Learning Science (2005)

12. Ilgen, D.R., Fisher, C., Taylor, M.S.: Consequences of individual feedback on behavior in organizations. Journal of Applied Psychology 64(4), 349-371 (1979)

13. Zander, A., Wolfe, D.: Administrative rewards and coordination among committee members. Administrative Science Quarterly 9, 50-69 (1964)

14. DeShon, R.P., Kozlowski, S.W.J., Schmidt, A.M., Milner, K.R., Wiechmann, D.: A multiple-goal, multilevel model of feedback effects on the regulation of individual and team performance. Journal of Applied Psychology 89(6), 1035-1056 (2004)

15. Cross, R., Borgatti, S.P., Parker, A.: Making invisible work visible: Using social network analysis to support strategic collaboration. California Management Review 44(2), 25-46 (2002)

16. Wellman, B.: For a social network analysis of computer networks: A sociological perspective on collaborative work and virtual community. In: Proceedings of the, ACM SIGCPR/SIGMIS Conference on Computer Personnel Research, pp. 1-11 (1996)

17. Park, H.W.: Hyperlink Network Analysis: A new method for the study of social structure on the Web. Connections 25(1), 49-61 (2003)

18. Donath, J., Boyd, D.: Public displays of connection. BT Technology Journal 22(4), 71-82 (2004)

19. Martino, F., Spoto, A.: Social Network Analysis: a brief theoretical review and further perspectives in the study of information technology. PsychNology 4(1), 53-86 (2006)

20. Isbell, C.L.I., Kearns, M., Kormannn, D., Singh, S., Stone, P.: Cobot in LamdaMOO: An adaptive social statistics agent. Autonomous Agents and Multi-Agent Systems 13(3), 327-354 (2000)

21. Morris, M.E.: Social networks as health feedback displays. IEEE Internet Computing 9(5), 29-37 (2005)

22. http://crossfire.real-time.com/

23. Borgatti, S.P., Everett, M.G., Freeman, L.C.: Ucinet for Windows: Software for Social Network Analysis. Analytic Technologies, Harvard, MA (2002)

24. Snijders, T.A.B., Borgatti, S.P.: Non-parametric standard errors and test for network statistics. Connections 22(2), 61-70 (1999) 